Meta

Journal des tradlucteurs

Translators' Journal

\title{
La métaphore dans la langue de la presse d'aujourd'hui
}

\section{K.-Richard Bausch}

Volume 13, numéro 4, décembre 1968

URI : https://id.erudit.org/iderudit/002009ar

DOI : https://doi.org/10.7202/002009ar

Aller au sommaire du numéro

Éditeur(s)

Les Presses de l'Université de Montréal

\section{ISSN}

0026-0452 (imprimé)

1492-1421 (numérique)

Découvrir la revue

Citer cet article

Bausch, K.-R. (1968). La métaphore dans la langue de la presse d'aujourd'hui. Meta, 13(4), 171-179. https://doi.org/10.7202/002009ar d'utilisation que vous pouvez consulter en ligne.

https://apropos.erudit.org/fr/usagers/politique-dutilisation/ 


\section{la métaphore dans la langue de la presse d'aujourd'hui}

1.0. Le concept de la métaphore a été considéré et défini de différents points de vue, divergeant les uns des autres ${ }^{1}$. Le critère sémantique de la transposition de signification par lequel, en général, deux champs de signification indépendants l'un de l'autre sont regroupés à un autre niveau, à savoir celui de la métaphore, est pourtant commun à toutes ces définitions.

C'est ainsi que, par exemple, la métaphore agonie de la $I V^{e}$ République allie les champs de signification de la médecine et de la politique et se détache de la simple dénomination indicative d'un état de choses: fin de la $I V^{e}$ République.

1.1. Nous savons qu'à plusieurs reprises, l'emploi de métaphores originales et surtout expressives a été mis en valeur comme caractéristique d'un style brillant et dense, mais exclusivement pour les textes littéraires et avant tout poétiques ${ }^{2}$. H. Konrad, par exemple, a mis en relief la différence entre la métaphore esthétique et la métaphore linguistique pied de la table, c'est-à-dire qu'il a opposé le moyen artistique individuel et motivé au signe arbitraire ${ }^{3}$.

1. Dans les œuvres linguistiques et littéraires, chez Quintilien pour la première fois probablement, la métaphore était considérée comme une sorte de comparaison abrégée (cf. entre autres, J. Marouzeau, Lexique de la terminologie linguistique, Paris, Genthner, 1951). Des auteurs plus récents, comme p. ex. E. Coseriu dans la Creación metatórica en el lenguaje, Montevideo, 1956, p. 16, ont donné des définitions différentes. Citons ici l'excellente discussion dans l'ouvrage de $\mathrm{H}$. Meier, Die Metapher. Versuch einer zusammenfassenden Betrachtung ihrer linguistischen Merkmale, Winterthur, 1963, p. 172 sqq., ainsi que la liste de 125 définitions du concept de la métaphore depuis Aristote jusqu'en 1963 de H. H. Lieb, Der Umfang des Metapherbegriffs (thèse), Cologne, 1964, p. 120 sqq.

2. Nous pourrions d'autre part interpréter la métaphore affective comme une caractéristique de la langue populaire ou vulgaire. De plus, nous devons considérer que la métaphore purement expressive aussi bien que la métaphore affective, en raison de leur caractère fortement subjectif, ne sont pas toujours compréhensibles au lecteur. Citons ici, également, les concepts de "métaphore affective " et de "métaphore explicative " chez H. Adank dans Essai sur les fondements psychologiques et linguistiques de la métaphore affective (thèse), Genève, 1939.

3. Dans Etude sur la métaphore (thèse), Paris, 1939. 
1.2. Au texte poétique, avant tout expressif, s'oppose le texte terminologique et à fin surtout informatrice, pour lequel c'est le caractère substantiel et non pas stylistique qui est prépondérant: l'emploi de métaphores consciemment présentes à l'esprit en général, ou même de métaphores insolites, uniquement expressives en particulier, freine le libre cours de la communication sobre, objective et concrète. L'une des caractéristiques des textes techniques, administratifs et juridiques est essentiellement leur manque de métaphores; ils sont donc spécifiquement $a$-stylistiques ${ }^{4}$.

Entre le groupe des textes informateurs et celui des textes expressifs s'insère le large éventail des genres restants. De ces genres mixtes de combinaisons à intensité différente peut être extrait le genre journalistique, divisé lui-même en politique, économique, etc. ${ }^{5}$. Le type journalisique est composé essentiellement d'une combinaison d'éléments clairement informateurs, mais, en même temps, tout aussi clairement stylistiques ${ }^{6}$.

La quantité même des métaphores utilisées nous amène à désigner la métaphore comme l'un des éléments stylistiques de la langue de la presse d'aujourd'hui ${ }^{7}$. La langue des journaux, type de langue commune, est généralement de composition spontanée: l'actualité informatrice du texte empêche, en effet, un long processus de formulation. La composition spontanée de l'ensemble du texte se reporte sur la nature des métaphores «spontanées» employées. Comme les «métaphores esthétiques », elles aussi sont caractérisées par leur nouveauté et leur originalité et, en général, ne font pas encore partie de la langue de tous les jours. Cependant, comme elles sont motivées par la nécessité, présente dans tout texte journalistique, d'attirer l'attention du lecteur, nous sous-entendons que l'aspect purement expressif est généralement perdu ${ }^{8}$.

4. Ce fait évident ne devra pas nous faire oublier que réciproquement les «terminologies» servent pour ainsi dire de réservoir métaphorique pour la langue commune (rappelons p. ex. les expressions du langage des chasseurs à un ou plusieurs éléments comme: ameuter, amorce, etc.; c'est là que gît le lièvre, revenir bredouille, etc.).

5. Il est extrêmement difficile de trouver dans une langue vivante, une classification globale pour les différents types de textes. Si certains schémas ont été établis, aucune solution satisfaisante n'a pourtant encore été trouvée.

6. Dans notre analyse, le concept de "style " n'est pas un terme de critique normative, mais de description linguistique. Nous ne considérons pas les problèmes du "bon " ou du " mauvais» style dans l'emploi ou dans la composition d'une certaine métaphore, nous nous intéressons exclusivement à la fonction stylistique de la métaphore. D'ailleurs, à notre avis, la qualification morale de "bon " ou de " mauvais " serait déplacée dans une analyse purement linguistique.

7. Nous pensons ici à la métaphore immédiatement intelligible, c'est-à-dire, à la métaphore dont l'emploi nous indique qu'un processus de transposition a lieu. Les métaphores pâlies - métaphores dites mortes comme p. ex.: le coucher du soleil; pied de la table, etc. c'est-à-dire les "métaphores linguistiques " seront négligées ici, car elles apparaissent dans tous les genres de textes, au même titre que les autres signes linguistiques. Voyons aussi W. Porzig, Das Wunder der Sprache, Berne-Munich, p. 40: «Das [die Lebendigkeit] gilt freilich nur solange, als die Sprechenden daran denken, wo die übertragenen Wörter eigentlich zu Hause sind. Tun sie das nicht mehr, so sind..., die Metaphern verblasst ".

8. Cf. le concept du Bildfeld (champ métaphorique) de $\mathbf{H}$. Weinrich d'abord dans son article "Münze und Wort. Untersuchungen an einem Bildfeld », dans Romanica; Festschrift $G$. Rohlfs, Halle, 1958 , p. 508 sqq., puis en résumé dans «Semantik der Metapher », dans Folia Linguistica; Acta Societatis Linguisticae Europeae vol. I, nos 1 et 2 La Haye, 1967 p. 12 sqq. (cf. en plus 2.0. sqq. de notre article). Selon la thèse de $H$. Weinrich, nous pourrions dire qu'une métaphore spontanée consiste en une combinaison originale d'un bildspendenden et d'un bildempfangenden Element dans son Bildfeld respectif (les termes techniques, cf. 1.2. de notre article ). 
Il faut pourtant constater: a) que seul un nombre faible de métaphores, créées pour les situations concrètes d'un texte, s'intègre dans le système linguistique comme élément de structure lexicale ${ }^{9}$; b) que la désignation d'une certaine expression par le terme de métaphore spontanée dépend de la relativité subjective, c'est-à-dire que l'emploi répété d'une métaphore, nouvelle à l'origine, entraîne l'usure; ainsi, dans certains cas particuliers, c'est l'appréciation linguistique personnelle qui décide de la spontanéité ou de la non-spontanéité ${ }^{10}$ (voir $\mathrm{p}$. ex. les métaphores usées, provenant partiellement du domaine de la tactique militaire: campagne antireligieuse; campagne en faveur de la contraception; offensive de charme; offensive de paix, etc.); c) que si, comme nous l'avons indiqué en b), la métaphore perd petit à petit de sa force, il ne reste à la fin de l'image originale qu'un signe arbitraire (voir note 7), c'est-à-dire une dénomination directe ou, selon Charles Bally, un homonyme dont le signifiant contient deux signifiés sans aucune relation intérieure (p. ex. la plume d'oiseau et la plume d'acier). Dans ce cas, nous dirons que la métaphore est morte.

1.3. Nous pouvons diviser selon la classification traditionnelle les métaphores en: a) métaphores lexicales, formées sur le plan du lexique; elles ne comportent généralement qu'un seul élément lexical ( $\mathrm{p}$. ex. homme-grenouille; pouls de la violence; mitraille d'informations);

b) métaphores syntaxiques, formées sur le plan de la syntaxe ${ }^{11}$; elles comportent plusieurs éléments lexicaux (p. ex. «... ce lancement permanent du produit révolution, celui qui lave le plus blanc pour nettoyer l'impérialisme américain »).

L'un des « traits pertinents » d'ordre général sur le plan syntaxique par rapport au plan lexical est la liaison au contexte, c'est-à-dire que des signes lexicaux «sans contexte » sont actualisés dans une certaine chaîne parlée. Nous dirons donc que le signe lexical est isolé, tandis que le groupe syntaxique existe grâce à la liaison de plusieurs signes linguistiques. Le contexte n'est donc typique que pour la syntaxe. Si nous reportons le critère du contexte à l'opposition traditionnelle: métaphore lexicale, métaphore syntaxique, il nous faut retenir que, contrairement au critère général dont nous parlions plus haut, la métaphore lexicale elle aussi ne devient métaphore que grâce au contexte. Les signes lexicaux grenouille, pouls, mitraille, que nous avons donnés en exemple, ne reçoivent leur signification métaphorique que par leur liaison étroite avec le contexte, donc avec homme, de la violence, d'informations. Ceci s'applique également à la métaphore syntaxique: par définition, celle-ci suppose une unité syntaxique du contexte celui qui lave

9. Cf. aussi Coseriu, op. cit., p. 16 sqq. H. Adank, dans son Essai sur les fondements psychologiques et linguistiques de la métaphore affective, a considéré la métaphore comme un "emprunt intérieur", c'est-à-dire, comme un processus qui, contrairement à l'emprunt de mots étrangers, a lieu au sein d'une même langue. Nous ajoutons à cette remarque que l'emploi d'un mot étranger signifie surtout un enrichissement de la langue d'arrivée, alors que la création d'une métaphore revient surtout à un enrichissement du texte (cf. 1.3. et note 12 de notre article).

10. Cf. entre autres, M. Bréal, Essai de sémantique: Science des significations, Paris, Hachette, 1904, p. 23: "Son succès même [de la métaphore] la fait pâlir."

11. Naturellement, la méthode diachronique nous montre la réduction d'une métaphore syntaxique en métaphore lexicale et réciproquement (voir p. ex. le composé rideau de fer et sa réactivation métaphorique dans rideau de fer épais). 
le plus blanc, mais elle aussi n'atteint la signification métaphorique transposée que par la liaison avec d'autres signes dans le contexte: révolution, impérialisme américain, etc.

La différence entre la métaphore lexicale et la métaphore syntaxique, en ce qui concerne le critère du contexte, est donc plutôt de nature quantitative que qualitative: toutes les deux ont besoin d'être liées au contexte. Par là cependant, elles appartiennent au domaine de la sémantique du texte et non pas à celui de la sémantique lexicale $^{12}$ (cf. 3.0. sqq.).

2.0. Après ces considérations générales que nous estimons indispensables à la classification ci-dessous, abordons à présent le côté pratique de notre analyse en reprenant le concept de «champ métaphorique » (Bildfeld) de H. Weinrich ${ }^{13}$. Depuis J. Trier, à côté de la classification syntagmatique des mots, la classification paradigmatique dans un champ sémantique a été l'objet de nombreuses analyses ${ }^{14}$. Nous pouvons établir des classifications paradigmatiques semblables pour les métaphores. Ainsi $\mathbf{H}$. Weinrich, par analogie consciente avec le champ sémantique de Trier, a introduit le concept du «champ métaphorique», dont il a donné la définition suivante: le champ métaphorique est l'accouplement de deux champs de signification par un acte spirituel, créant une analogie. C'est donc la liaison de deux champs sémantiques en un seul champ métaphorique, dans lequel l'un des champs de signification devient le champ émetteur d'une image tandis que le second devient le champ récepteur d'une image ${ }^{15}$.

Dans une première partie, nous classerons les exemples de métaphores à notre disposition en champs métaphoriques et, dans une deuxième partie, nous exposerons les fonctions stylistiques de quelques métaphores dans la langue de la presse d'aujourd'hui ${ }^{16}$.

12. Voir aussi Weinrich, "Semantik..." " p. 5: "Weniger elementar wird dann allerdings schon die Beobachtung sein, dass Metaphern, im Unterschied zu Normalwörtern, unter keinen Umständen von den Kontextbedingungen entbunden werden können... Wir haben daher die Wortsemantik notwendig zu einer Textsemantik hin zu überschreiten. (Und es würe ein grosser Irrtum zu glauben, Textsemantik sei dasselbe wie Syntax.) 》Voir, en outre, l'opposition "langue-parole " de F. de Saussure, chez E. Coseriu, "Determinación y entorno", dans Teoria del lenguaje y lingïistica general, Madrid, 1962, p. 293: "Naturalmente, una metáfora se reconoce como tal en la medida en que ambos valores (el "nombrado" y el "denotado") se perciben al mismo tiempo como diversos y como asimilados. También el tema de la metáfora pertenece, pues, a la lingǘística del hablar."

13. Ses travaux sont cités dans la note 8 .

14. Voir J. Trier, surtout Der deutsche Wortschatz im Sinnbezirk des Verstandes. Die Geschichte eines sprachlichen Feldes, Heidelberg, 1931.

15. Voir Weinrich, "Münze... ", p. 514, et en résumé "Semantik... ", p. 13. Le concept de champ métaphorique a des précurseurs, comme p. ex. K. Bühler, Sprachtheorie. Die Darsiellungsfunktion der Sprache, Iéna, 1934, qui, pour la métaphore der greise Wald, parle d'un recoupement, d'un engrenage des sphères d'imagination de l'humain, greis et de la nature, Wald.

16. Ces deux parties sont fondées sur une documentation d'environ 500 métaphores extraites des journaux et périodiques suivants: le Monde, 8 juin 1967 (M 1), 16-17 juin 1967 (M 2), 18 juin 1967 (M 3), 19 juin 1967 (M 4); France-Soir, 12 août 1967 (FS 1), 13-14 août 1967 (FS 2), 15 août 1967 (FS 3), 16 août 1967 (FS 4); l'Express, 7-13 août 1967 (E 1), 14-20 août 1967 (E 2), 21-27 août 1967 (E 3), 28 août-3 septembre 1967 (E 4); le Nouvel Observateur, 27 août-3 septembre 1967 (O 1), 11-17 septembre 1967 (O 2), 20-26 septembre 1967 (O 3) 4-10 novembre 1967 (O 4). Les métaphores se trouvaient essentiellement dans les articles politiques, économiques et ceux qui concernaient les affaires sociales. Sous notre direction, $\mathbf{M}^{\text {lle }}$ Sigrun Rainders a compilé et interprété 
2.1. Nous nous contenterons des champs récepteurs de la politique et de l'économie ${ }^{17}$ auxquels s'opposent surtout les champs émetteurs comme la médecine, la stratégie militaire, le théâtre, le jeu, la circulation et la météorologie. Nous établirons ainsi, selon la fréquence de leur usage, les six champs métaphoriques suivants ${ }^{18}$ :

1. Le champ de la médecine politique; il se subdivise en:

a) remèdes politiques

La guerre du Vietnam a été le meilleur antidote de l'économie américaine. (E 1, p. 27)

Le gouvernement mitonne pour l'automne quelques calmants. (E 1, p. 9)

Les gouvernements injectent des crédits. (E 1, p.9)

b) maladies politiques

L'assoupissement de l'administration. (E 3, p. 18)

Les Français, malgré leur désir, ont du mal à échapper à cette fièvre collective. (FS 2, p. 4) Une fièvre maligne [de l'économie française] qui allait tomber toute seule, sans qu'il soit nécessaire de lui administrer des remèdes de cheval. (E 1, p. 9)

C'est une opération d'intoxication américaine. (O 1, p. 4)

Les allergies idéologiques. (O 4, p. 23 )

Les périphéries lépreuses des métropoles. ( 04, p. 4)

Des entorses graves, apportées aux libertés fondamentales de la société américaine. $(\mathrm{M} 4$, p. 3$)$ Le prince Rainier, de passage à Vancouver, a posé son demi-grain de sel sur la plaie canadienne. (E 1, p. 6)

2. Le champ de la stratégie militaire politique; il se subdivise en:

a) actions militaires politiques et théâtre de la guerre

Les fonctionnaires bombardent de notes les directeurs d'usine. (E 4, p. 22)

Prendre d'assaut certains bastions de la spéculation et du malthusianisme. (O 1, p. 9)

Torpiller un projet soviético-américain. (E 2, p. 20)

... M. Schröder, qui aurait ainsi posé une mine à retardement pour mieux défendre son budget. (M 4, p. 1)

Ces salves de contre-artillerie venaient peut-être trop tard... la fronde, progressivement, a succédé au doute. (E 4, p. 4)

M. Mitterand se sent à la porte du pouvoir; mais il sait qu'il lui manque encore, pour y accéder, une fraction de l'électorat et un détonateur dans le camp adverse. (E 1, p. 17)

Une mitraille d'informations. (M 3, p. 15)

b) tactique de guerre politique

Le style Garde-Rouge. (E 2, p. 19)

Dans cette atmosphère de guerre froide psychologique les stratèges de la diplomatie israélienne se battent sur quatre fronts. (E 4, p. 20)

Dernière des fausses manceuvres, l'affaire des anciens... (E 3, p. 9)

Ni sur cette stratégie de fond, ni sur cette méthode tactique le discours tactique du... (E 2, p. 7)

ces métaphores avec beaucoup d'application et de sagacité dans son mémoire présenté à l'Institut fuer Uebersetzen und Dolmetschen de l'Université de la Sarre. Nous tenons à lui exprimer tous nos remerciements.

17. Comme dans la réalité extra-linguistique ces deux domaines sont étroitement liés, il n'est pas étonnant qu'au sein des champs métaphoriques ils aient en grande partie les mêmes champs émetteurs d'images. Si, dans ce qui suit, pour simplifier nous ne parlons que de politique, il est évident que ce concept comprend également des exemples appartenant à l'économie.

18. Une affirmation aussi peu appuyée du point de vue quantitatif est contestable. Nous sommes conscients aussi du fait que nous n'avons pas atteint la totalité des possibilités. Ainsi, p. ex.: les champs "sport", "technique", etc.; cf. Meier, op. cit., p. 195 sqq. 
3. Le champ du théâtre politique ${ }^{19}$; il se subdivise en:

a) scène politique

Pour un peuple qui en a une très vieille habitude, il est pénible de quitter la scène pour passer du côté des spectateurs. (E 3, p. 23)

Les deux ordonnances majeures viennent clopin-clopant d'entrer sur la scène nationale. (E 1, p. 4)

Débats, plus officieux, aussi dans les couloirs, dans les coulisses. (O 1, p. 12)

b) acteurs, figurants politiques

Dans un bon numéro d'acteur, le Général s'est battu pour ressusciter une magie. (E 2, p. 4) L'Inde non violente joue aujourd'hui le rôle de va-t-en guerre. (M 4, p. 4)

On croit savoir que les autorités algériennes considèrent pour le moment les trois Belges comme des comparses. (M 2, p. 5)

c) genres politico-littéraires

La comédie du désarmement. (E 4, p. 18)

Le mélodrame canadien. (E 2, p. 4)

Un des drames du Ghetto. (E 2, p. 22)

Le show canadien. (E 2, p. 4)

4. Le champ du jeu politique; il se subdivise en:

a) jeu de cartes politique

De Gaulle, dès le premier soir, abattit ses cartes. ( $(02, \mathrm{p} .3)$

Le groupe qui parviendra à s'emparer de Pétrofina mettra un atout maître dans ses jeux.

(O 1, p. 12)

Jouer la carte de la paix au Vietnam. (O 2, p. 8)

b) jeu d'échecs politique

... utilisant imperturbablement toutes les failles du système pour pousser ses pions. $(M 4, \mathbf{p} .1)$

Et, pour l'heure, ni M. François Mitterand ni M. Giscard d'Estaing, absorbés dans la partie, où il s'agit de savoir lequel des deux fera échec et mat au roi... (E $3, \mathrm{p} .6)$

c) différents jeux politiques

Les experts occidentaux rendent des sentences. Ils jouent au jeu des caramels. $(04, \mathrm{p} .5)$

Mener un jeu de bascule entre gaullistes et giscardiens. $(04$, p. 15)

Il y a d'abord un carrousel de ministres dans le pays. $(\mathrm{O} 3, \mathrm{p} .13)$

5. Le champ de la circulation politique; il se subdivise en:

a) réseau de circulation politique

Les avenues du pouvoir. (E 2, p. 4)

La voie de la catastrophe. (FS 3, p. 4)

De toute façon, maintenant le tournant est pris: Amer disparu, Nasser se retrouve seul...

(O 3, p. 14)

Déviations soviétiques du socialisme. (O 2, p. 7)

Carrefour intellectuel. (M 3, p. 14)

Feu vert pour le rachat de... (E 3, p. 22)

Priorité au tourisme. (E 3, p. 20)

19. Voir aussi Meier, op. cit., p. 200: "Das Theater mit den ihm zugehörigen Begriffen ist an sich zwar kein neuer Vorstellungskomplex. Dessen ungeachtet, zeigt vor allem die moderne Journalistik eine ausgesprochene Vorliebe für Ubertragungen aus der Welt des Theaters, und wenigstens einige davon dürften zweifellos neu sein." 
b) moyens de communication politiques

Voici le moment du coup de volant inévitable. (M 3, p. 6)

Les clignotants ont failli s'allumer. Faudra-t-il reviser le $\mathrm{V}^{\mathrm{e}} \mathrm{Plan}$ en cours de route ? (E 2, p. 8)

L'embrayage entre cette manifestation de volonté... (O 2, p. 7)

6. Le champ de la météorologie politique

Le baromètre est donc serein en ce qui concerne la politique étrangère; il affiche plutôt variable dans le domaine intérieur. (M 4, p. 1)

La température des relations entre le Nord-Vietnam et la Chine. (E 2, p. 18)

Les variations du climat politique au Moyen-Orient. (M 4, p. 1)

Après quelques heures de débats orageux. (M 1, p. 1)

M. Boudan a parlé de louragan qui a emporté un tiers des préfets des départements français. (M 2, p. 5)

Il y a des nuages entre Pompidou et de Gaulle. (O 2, p. 4)

3.0. Par définition, la métaphore a une fonction d'illustration. En même temps, elle n'exprime pas seulement le pourquoi ou le comment d'une chose, mais elle tente d'obtenir un certain effet (cf. l'exemple de 1.0.: agonie de la IVe République et la simple dénomination fin de la $I V^{e}$ République).

Ces effets de style semblent s'appliquer dans une forte mesure aux métaphores spontanées, non rebattues, qui deviennent par là le moyen de style par excellence. En considérant la documentation dont nous disposons, nous voyons que, dans la langue de la presse, la métaphore remplit essentiellement les fonctions stylistiques suivantes ${ }^{20}$ :

1. Comique

C'est comme la mini-jupe qui a choqué au début. Il nous faut prendre l'habitude. Giscard dit: "Voyez, $j^{\prime}$ ai de jolies jambes *. Pompidou doit à son tour montrer les siennes! (O 2, p. 5)

Dans cette métaphore liée à une comparaison explicite, la campagne électorale est menée comme un concours de beauté. L'effet prépondérant du comique est évident ${ }^{21}$.

La propagande [à Cuba] est intense, obsédante, fascinante. La plus agressive des campagnes publicitaires pâlirait en face de ce lancement permanent du produit révolution, celui qui lave le plus blanc pour nettoyer l'impérialisme américain. (E 1, p. 22)

On a ici un mélange des domaines de la publicité et de la politique: la révolution est considérée comme slogan publicitaire du nettoyage, l'impérialisme comme celui de la saleté. Ce mélange original provoque un effet comique.

Les adieux de Castro et de Che Guevara sont représentés de la façon suivante: Castro tempête, s'émeut, se laisse convaincre. Rideau sur la scène shakespearienne. $(\mathrm{O} 4, \mathrm{p} .8)$ Les syndicats français donnent le commentaire suivant des mesures sociales de de Gaulle en été 1967:

Quand la potion a été prête, on a mis dedans le morceau de sucre de l'intéressement. (E 1, p. 6)

20. Pour cette présentation stylistique, les différents exemples sont isolés de leur champ métaphorique et ne sont considérés que dans leur contexte respectif. La différence n'est pas faite entre les métaphores lexicales et les métaphores syntaxiques. Le classement a encore été établi selon le critère de la fréquence d'emploi (cf. note 18).

21. Bien entendu nous ne voulons pas par là éliminer les autres effets stylistiques "moindres » (ainsi dans la métaphore qui suit, il y a sans doute, à côté de l'effet comique, une certaine concrétisation stylistique (cf. produit révolution, etc.). Cependant l'effet que nous avons choisi est caractéristique et prévaut ici aussi bien que dans les autres groupes. 
Les mesures prises par de Gaulle paraissent «amères » au premier abord et sont ensuite "adoucies》 par l'intéressement des travailleurs.

Le rachat d'une fabrique de biscuits française par une société américaine, la General Mills, fut l'occasion de la métaphore comique suivante:

Pour que le grand méchant loup américain ne le dévore pas, le petit biscuit français a appelé à laide son grand frère de l'industrie alimentaire. (E 1, p. 22)

2. Ironie

L'effet comique et l'effet ironique peuvent être très proches l'un de l'autre comme le prouve la recette culinaire ou, si l'on veut, recette de cocktail métaphorique suivante:

Vous choisissez les « conditions objectives » favorables, le moment propice et le lieu opportun, en l'occurence la forêt bolivienne. Vous prenez des militants communistes pro-soviétiques, communistes pro-chinois, trotskistes, nationaux révolutionnaires, pour que chaque courant politique se sente représenté et intéressé. Vous ajoutez une pincée, un levain d'intellectuels commissaires politiques et médecins. Vous mélangez à proportions égales - c'est tout le secret de la recette... Vous agitez, vous agitez beaucoup. Vous servez chaud, très chaud. (O 4, p. 9)

De Gaulle, "insoucieux de tous les équipages », chemine, lui, du Saint-Laurent à l'Oder-Neisse, réveillant les nationalismes du siècle passé. (E 3, p. 7)

Dans le Bateau ivre de Rimbaud nous lisons: «j'étais insoucieux de tous les équipages $\gg$. En plaçant les guillemets à dessein, l'analogie avec le Bateau ivre est établie. Cela cherche à souligner l'attitude peu réaliste et égocentrique du Général, capitaine du vaisseau qu'est l'État ${ }^{22}$.

3. Concrétisation

L'ambiance politique en Roumanie est exprimée par les mesures concrètes du baromètre:

Le baromètre est donc serein en ce qui concerne la politique étrangère, il affiche plutôt variable dans le domaine intérieur. (M 4, p. 1)

De Gaulle refuse de répondre aux provocations de Giscard d'Estaing dans une confrontation franche:

Mais le Général de Gaulle se gardait bien de descendre dans l'arène. (O 2, p. 4 )

Le rideau de fer tsariste était déjà assez épais pour son temps, même si le Kremlin a fait beaucoup mieux depuis. (M 4, p. 3)

La métaphore rideau de fer a perdu aujourd'hui beaucoup de son caractère figuré. Elle fait partie des métaphores «pâlies» ou «mortes». L'usage fréquent y fut pour une grande part. Grâce à la caractérisation par l'adjectif épciss, le rideau redevient «matériel » et ainsi la métaphore est concrétisée et ravivée (comparer avec la note 11).

4. Contraste insolite (Verfremdung)

L'effet stylistique de la Verfremdung provient du mélange particulièrement insolite de deux domaines de signification dans la métaphore:

Par exemple, comme le montre l'extrait suivant, lorsque la personnalité politique de de Gaulle est liée à la doctrine chrétienne du salut:

22. Généralement pourtant les signes typographiques prouvent qu'il s'agit de métaphores peu usitées que l'auteur veut caractériser en tant que telles ( $\mathrm{p}$. ex.: "Vote presse-bouton en octobre à l'Assemblée. " (France-Soir, 12 avril 1958) "... le général Nicot aurait courtcircuité les ordres... "(France-Soir, 4 mai 1961) cité d'après Meier, op. cit., p. 217. 
On approuve de Gaulle en tout et l'on est alors avec lui sur la voie du salut, ou bien, en étant réservé sur tel ou tel point particulier, on montre une capacité à saisir que sa politique forme un tout cohérent et l'on se range du côté des ilotes ou des Judas. (E 2, p. 7)

Ce continent [l'Amérique du Sud] porte depuis longtemps une révolution dans ses entrailles, et ce n'est pas l'absence des conditions objectives qui a retardé son accouchement. (O 3, p. 19) À l'aide des métaphores entrailles et accouchement la révolution en Amérique $\mathrm{du}$ Sud est dite grandie et portée à terme dans le « corps vivant » du continent. La politique gaulliste se déplo:e à l'intérieur d'une alliance de fait des deux mastodontes et lui donne à lui, de Gaulle, des possibilités de jeu entre leurs énormes pattes. (M 3, p. 6)

Dans cette métaphore les deux super-grands apparaissent comme les monstres de «la préhistoire politique ». La liberté d'action politique de de Gaulle est réduite à un jeu inoffensif.

La Verfremdung devient dramatique lorsque, à propos de l'abolition des barrières douanières au sein du Marché commun (abolition considérée comme un danger menaçant l'industrie française), on évoque la Révolution française:

Cela dit, le couperet ne va pas tomber comme cela, d'un seul coup, le $1^{\text {er }}$ juillet 1968.23 (E 2, p. 9)

4.0. Depuis Aristote, maintes tentatives ont été faites en vue de classer les métaphores, chaque fois sous un aspect nouveau ou différent. Les textes littéraires en étaient les sources presque uniques. Il est pourtant incontestable aujourd'hui que la métaphore n'est plus le privilège exclusif du texte élaboré et formulé avec le plus grand soin. Nous avons vu qu'elle est en même temps l'une des caractéristiques essentielles de la langue spontanée et éphémère de la presse d'aujourd'hui. Son efficacité essentielle réside dans le fait que la transposition métaphorique de concepts de la vie quotidienne (comme p. ex. le jeu, la circulation, etc., qui concernent le lecteur directement) fait pénétrer les événements impersonnels et indirects de la politique, de l'économie, etc. dans un champ d'expérience pour lui plus direct et plus compréhensible.

K.-RICHARD BAUSCH

23. Cette liste d'interprétations peut, bien entendu, être complétée par d'autres exemples sans la moindre difficulté. 